\title{
On Health Policy and Management (HPAM): mind the theory-policy-practice gap
}

\author{
David P Chinitz ${ }^{1, *}$, Victor G Rodwin ${ }^{2}$
}

\begin{abstract}
We argue that the field of Health Policy and Management (HPAM) ought to confront the gap between theory, policy, and practice. Although there are perennial efforts to reform healthcare systems, the conceptual barriers are considerable and reflect the theory-policy-practice gap. We highlight four dimensions of the gap: 1) the dominance of microeconomic thinking in health policy analysis and design; 2) the lack of learning from management theory and comparative case studies; 3 ) the separation of HPAM from the rank and file of healthcare; and 4) the failure to expose medical students to issues of HPAM. We conclude with suggestions for rethinking the field of HPAM by embracing broader perspectives, e.g. ethics, urban health, systems analysis and cross-national analyses of healthcare systems. Keywords: Health Policy, Health Management Theory, Medical Education

Copyright: $\odot 2014$ by Kerman University of Medical Sciences

Citation: Chinitz DP, Rodwin VG. On Health Policy and Management (HPAM): mind the theory-policy-practice gap. Int J Health Policy Manag 2014; 3: 361-363. doi: 10.15171/ijhpm.2014.122
\end{abstract}

Article History:

Received: 28 September 2014 Accepted: 9 November 2014 ePublished: 11 November 2014

*Correspondence to:
David P Chinitz
Email: chinitz@cc.huji.ac.il
$H$ ealth Affairs editor, Alan Weil (1), notes that "The path to effective public policy is rarely straight. Odds are low that we will get it right on our first attempt to address a need or solve a problem. Usually, the best we can hope for is rapid understanding and recalibration of our policies as we begin to see their positive and often unintended negative effects". Weil assumes that problems can eventually be solved and the solutions implemented. However, the field of Health Policy and Management (HPAM) must more often tackle "wicked problems" (2) that are uniquely affected by their institutional contexts and whose potential "solutions" are shaped by the ways in which they are formulated. Thus, in contributing to "effective policy" and management, we argue that it is also important for policy analysts and managers to mind the gap between theory, policy and practice in the field of HPAM. Despite decades of efforts aimed at reforming healthcare systems in the United States, little has changed in the basic arrangements within which physicians practice. The U.S. continues to have fragmented healthcare systems that shun vertical integration across hospitals and communitybased primary care. Porter and Lee (3) state that "countless incremental changes" in the U.S. have not had much impact and propose a new strategy: "the value agenda". While we agree, we fear that such a new strategy, as evidenced in the ambivalent literature about Accountable Care Organizations (ACOs), Health Information Technology (HIT), Pay-forPerformance (P4P) and Value-Based Purchasing will also fail and here we explain why and what to do about it. As we have discussed elsewhere at greater length (4), four problems sustain a theory-policy-practice gap that frustrates attempts to reform healthcare systems.

The dominance of microeconomic thinking in heath policy analysis and design

Prominent economists, themselves, have noted the dominance, and sometimes the overreach, of their discipline in social policy. One need only refer to Arrow's (5) classic article on healthcare to note the emphasis not only on information asymmetries leading to market failure, but also to the critical importance of trust in healthcare transactions. Hirschman's (6) seminal analysis of voice and loyalty in organizations highlights the limits of conventional market models that rely on "exit" in social systems, and the role of consumers to avoid the corrosive effects of market behavior. Despite these amendments to conventional economic models, health policy returns cyclically to financial incentives and market mechanisms as solutions to health systems that cost too much and provide too little.

The lack of learning from management theory and comparative case studies

Despite knowledge of how healthcare organizations work, management experts have far less influence on policy than economists. Much of healthcare management knowledge is derived from case studies - typically suspect in policy-making settings that seek to build theory on the basis of which policy changes may be justified. It is difficult to come up with general conclusions based on case studies of different organizations. A key tenet of organizational behavior and management is the contingency approach, namely, the insight that optimal behavior depends on local conditions and that there is no one best way to manage. For management experts this is not an easy position to defend when policy-makers prefer advisors who know the "invisible hand", in theory, and rarely say "but, on the other hand".

This predicament can be seen in the large number of healthcare management approaches that emphasize the importance of "culture". For example, Geisinger in Pennsylvania, Kaiser Permanente in California, Intermountain Health in Utah and the Mayo Clinics in 
Minnesota, Arizona, Iowa and Florida, are often touted as having high-performing healthcare systems. Their methods are sometimes even transferred to other settings, but there has been little success in generalizing such models across the U.S. The experience of "high performance" organizations has not yet yielded a body of wisdom on which to transform other healthcare organizations.

How to learn from case studies of individual healthcare systems remains poorly understood. There are more case studies written by "believers" in particular systems than systematic comparisons with common metrics, across different systems, that could capture the impact of local contingencies on the application of ideas from management theory. Leaders in the field of HPAM have sought inspiration from evidence-based medicine that involves horizontal comparison of medical interventions, to promote notions of evidence-based management. But organizational learning must also be vertical, i.e. based on information gathered from within the organization as opposed to looking elsewhere for lessons. Trying to combine these horizontal and vertical learning approaches wilts in the face of pressure to produce short-term payoffs, and the impatience with slower knowledge accumulation based on case studies.

The separation of Health Policy and Management (HPAM) from the rank and file of healthcare

Beyond the dominance of microeconomic thinking and the lack of learning from management theory and comparative case studies of healthcare organizations, another problem that sustains the theory-policy-practice gap is the separation of HPAM from the rank and file of healthcare. Healthcare delivery organizations are often designed without sufficient participation from the rank and file, especially physicians. Their limited participation strikes us as inappropriate given their critical role in the provision of quality healthcare but is not surprising since the training and socialization of medical professionals is distant from considerations of cost, quality, and access. Although prevailing opinion in the field of HPAM suggests that regulation rooted in, for example, quality measurement, will eventually make key stakeholders come around (7), this approach has not worked so well (8). Despite ebbs and flows in managed care, grouping and regroupings of hospitals and physicians and decades of policy announcements calling for healthcare to become more effective and for the allocation of resources to become more efficient, and equitable, little has changed in the basic arrangements within which physicians practice.

While policy commentaries and perspective pieces in JAMA and NEJM promote ideas from the field of HPAM, rank-andfile medical professionals often find them removed from an understanding of what clinicians and managers face in the world of practice. Dominant HPAM approaches focus on technical tools for measuring, monitoring and comparison of the performance of health delivery systems. Such approaches neglect the complex internal workings of healthcare systems, about which rank and file physicians and nurses are the most knowledgeable. The result is to drive a wedge between the HPAM discussions going on in the intellectual and policy stratosphere and what is actually happening on the ground.
The failure to expose medical students to issues of Health Policy and Management (HPAM)

Medical education has given short shrift to HPAM because it does not train physicians to think about population health, quality assurance, and alternative institutional contexts within which medicine is practiced. Health policy is driven by a desire to control costs, assure equitable access to healthcare and improve service quality. Medical education resists change because there is simply too much to learn about how to treat individual diseases to divert medical students' attention to other matters. Medical students should, of course, be trained to treat individual patients. But imagine if they were also exposed to studies of variations in medical practice (9) and introduced to case studies of integrated team care without being told that there is a one best way of managing every patient pathway. Such broadening of medical education would - at the very least-sensitize medical professionals to concepts and pressures that they will surely confront in their professional practice.

\section{Rethinking Health Policy and Management (HPAM)}

In summary, a shift in how health policy analysts and managers think about health systems is long overdue and could narrow the theory-policy-practice gap. We suggest that the field of HPAM must be broadened and deepened so that public policy and management interventions draw more heavily from theory and policy that more closely capture the complexity and conflicts embedded within management and healthcare practices. The dominance of microeconomic theory must be challenged, comparative studies of healthcare organizations must be encouraged, participation of rank and file healthcare providers must be extended, and medical students must be introduced to issues of HPAM.

In broadening HPAM, it will be necessary to improve understanding of how financial incentives interact with professional values and organizational cultures. Beyond microeconomics, institutional economics, organization theory and management, HPAM must embrace (rather than shun) disciplines ranging from sociology, anthropology, epistemology as well as broader perspectives, e.g. ethics, urban health, systems analysis, and cross-national analyses of healthcare systems. There is a role for microeconomic thinking. But how such tools as ACOs, P4P, HIT are implemented ought to be studied with regard to how they might be adapted in different institutional contexts. And how they interact with professional values and norms should also be assessed so that refinements can be made to avoid crowding out positive behavior rooted in values other than pecuniary incentives.

\section{Ethical issues}

Not applicable.

Competing interests

Authors declare that they have no competing interests.

Authors' contributions

Both authors equally contributed to the writing and revision of this paper.

\section{Authors' affiliations}

${ }^{1}$ Hebrew University-Hadassah, Jerusalem, Israel. ${ }^{2}$ Wagner School of Public Service, New York University, New York, NY, USA. 


\section{References}

1. Weil AR. The winding path to effective policy. Health Aff (Millwood) 2014; 33: 1310. doi: 10.1377/hlthaff.2014.0799

2. Rittel H, Webber M. Dilemmas in a General Theory of Planning. Policy Sci 1973; 4: 155-69. doi: 10.1007/bf01405730

3. Porter ME, Lee TH. Why Health Care is Stuck- And How to Fix It [internet]. [updated 2013 Sept 17]. NEMJ/HBR Blog Network. Available from: https://hbr.org/2013/09/why-health-care-is-stuckand-how-to-fix-it/

4. Chinitz D, Rodwin VG. What passes and fails as health policy and management. J Health Polit Policy Law 2014; 39: 1113-26. doi: 10.1215/03616878-2813719

5. Arrow KJ. Uncertainty and the Welfare Economics of Medical Care. Am Econ Rev 1963; 53: 941-73.
6. Hirschman AO. Exit, Voice, and Loyalty: Responses to Declines in Firms, Organizations, and States. Cambridge, MA: Harvard University Press; 2013.

7. Dixon J, Chantler C, Billings J. Competition on outcomes and physician leadership are not enough to reform health care. JAMA 2007; 298: 1445-7. doi: 10.1001/jama.298.12.1445

8. Berenson RA, Pronovost PJ, Krumholz HM. Achieving the Potential of Health Care Performance Measures [internet]. Urban Institute. 2013. Available from: http://www.rwjf.org/en/ research-publications/find-rwjf-research/2013/05/achieving-thepotential-of-health-care-performance-measures.html

9. Wennberg JE. Dealing with Medical Practice Variations: A Proposal for Action. Health Aff (Millwood) 1984; 3: 6-32. doi: 10.1377/hlthaff.3.2.6 